\title{
Genome Sequence Analysis of Cronobacter Phage PF-CE2 and Proposal of a New Species in the Escherichia Virus RB16 Genus
}

\section{Mengshi Xiao}

Ocean University of China

\section{Xinmiao Ren}

Ocean University of China

\section{Ying Yu}

Ocean University of China

\section{Han Sun}

Ocean University of China

Haijin Mou ( $\triangle$ mousun@ouc.edu.cn )

Ocean University of China https://orcid.org/0000-0003-3667-1562

\section{Xiaodan Fu}

Ocean University of China

\section{Research Article}

Keywords: Cronobacter sakazakii, Escherichia virus, subsequent heterologous, PF-CE2 glycanase

Posted Date: June 21st, 2021

DOl: https://doi.org/10.21203/rs.3.rs-258819/v1

License: (c) (i) This work is licensed under a Creative Commons Attribution 4.0 International License. Read Full License 


\section{Abstract}

The genome of Cronobacter sakazakii M1 phage named PF-CE2 was characterized in this work. And a new species named Cronobacter virus PF-CE2, in the Escherichia virus RB16 genus of the subfamily Tevenvirinae of the family Myoviridae was established. The Gp190 gene of phage PF-CE2 was first proposed to encode a bacteriophage-borne glycanase, which is capable of degrading fucose-containing exopolysaccharides produced by $C$. sakazakii M1. Further, the taxonomic status of eight additional phages was modified according to average nucleotide identity analysis. This finding provides a theoretical basis for subsequent heterologous expression of the phage PF-CE2 glycanase and provides an important reference for the preservation and sharing of these phages.

\section{Main Text}

Cronobacter sakazakii are facultative, anaerobic Gram-negative bacteria that exist widely in various foods and raw materials $[1,2]$. In recent years, as a new foodborne pathogen, C. sakazakii has been commonly found in formula milk powder and is associated with several infectious diseases, including meningitis, necrotizing enterocolitis, and sepsis [3, 4]. Viruses are ubiquitous in nature, and bacteriophages, which are viruses of bacteria, are effective tools to kill pathogenic bacteria [5]. The genetic diversity of $C$. sakazakii poses a challenge for the use of phages to control microbial contamination in food processing environments, therefore it is necessary to isolate and identify new phages targeting C. sakazakii [6].

Fucose-containing exopolysaccharides (FcEPSs) are a promising source of fucosylated oligosaccharides and fucose. Cronobacter sp. typically have the capacity to produce fucose-rich FcEPSs [7]. Bacteriophage-borne glycanases extracted from phages are effective tools for degrading FcEPSs [8]. A previous study found that a phage isolated from sewage was capable of degrading FcEPS produced by C. sakazakii M1 [9]. In this study, the bacteriophage targeting $C$. sakazakii M1 was purified via a plaque assay, more than 10 times. The genome sequence and functional biological characterization of phage PF-CE2 was completed and compared with those of homologous phages. In addition, the gene encoding the bacteriophage-borne glycanase was predicted.

Phage isolation and purification were performed as described previously [9]. Briefly, prior to phage PF-CE2 DNA isolation, DNase I (10 $\mathrm{gg} / \mathrm{mL}$, Sigma-Aldrich) and RNase A (20 $\mu \mathrm{g} / \mathrm{mL}$, Sigma-Aldrich) were added to a purified suspension of PF-CE2 and incubated at $37^{\circ} \mathrm{C}$ for $1 \mathrm{~h}$, to digest bacterial DNA and RNA. DNA isolation and purification of phage PF-CE2 were carried out using the E.Z.N.A ${ }^{\circledR}$ Viral DNA Kit (OMEGA). The sequencing library was generated using the Illumina TruSeq DNA Nano Sample Preparation Kit (Illumina). One microgram of DNA was sheared into 300-500 bp fragments using M220 
Focused-ultrasonicator (Covaris). Following PCR amplification, the purpose straps were recovered by gel excision. A TBS-380 Micro-Fluorometer (Turner BioSystems) and PicoGreen ${ }^{\circledR}$ (ThermoFisher Scientific) were used for quantitative analysis and clusters were generated by bridging PCR amplification on cBot 2 system (Illumina). The genome of phage PF-CE2 was sequenced using the Illumina HiSeq system with a $2 \times 150$ bp pairedend run. To make sure the accuracy and reliability of the sequencing results, quality control of the original data was performed as follows: (1) removal of the adapter sequence from reads, (2) reads containing non-AGCT at the 5' end were removed, (3) the ends of reads whose sequencing quality was less than Q20 were removed, (4) reads whose $\mathrm{N}$ proportion was more than $10 \%$ were removed, and (5) fragments less than 50 bp were discarded. Following quality control, clean data were obtained; detailed information is shown in Table S1. The reads were assembled using ABySS (v2.0.2) assembly software, and GapCloser (v1.12) software was used to carry out gap filling and base correction. The results of high-throughput sequencing showed that phage PF-CE2 was assembled at 88-fold coverage into a genome of $178248 \mathrm{bp}$ in length, with a G + C content of $44.8 \%$ and that genes made up $95.87 \%$ of the genome. The genome sequence of phage PF-CE2 was compared with that of other phages using the standard nucleotide BLAST in the NCBI database (https://blast.ncbi.nlm.nih.gov/Blast.cgi). Table S2 shows the basic characteristics of eight selected phages, which are similar to phage PF-CE2 in length and $\mathrm{G}+\mathrm{C}$ content, including the Citrobacter phages (Margaery (unpublished) and Maroon [10]), Cronobacter phages (vB CsaM Cronuts (unpublished), vB CsaM GAP161 [11], vB CsaM leB [1], vB CsaM leE [1], and vB CsaM leN [1]), and Enterobacter phage vB EkoM5VN (unpublished). tRNAscan-SE 2.0 (http://lowelab.ucsc.edu/tRNAscan-SE/) was used to identify possible tRNAs in the genome, and rRNA was predicted using the RNAmmer 1.2 Server (http://www.cbs.dtu.dk/services/RNAmmer/) [12, 13]. None of them contained rRNA. tRNA ${ }^{\text {Met }}$ and tRNA ${ }^{\text {Gly }}$ were found in the genomes of PF-CE2,

Cronobacter phages vB CsaM Cronuts and vB CsaM GAP161, while the others contained only tRNA ${ }^{\text {Gly }}$. Comprehensive Antibiotic Resistance Database (https://card.mcmaster.ca/) and Virulence Factor Database (http://www.mgc.ac.cn/VFs/blast/blast.html) analyses did not detected antibiotic resistance encoding genes, or virulence factors in the genomes. A total of 275 open reading frames (ORFs) were identified using the ORF finder (https://www.ncbi.nlm.nih.gov/orffinder/), of which 123 were located on the plus strand and the remainder on the minus strand (Table S3). Blastp (https://blast.ncbi.nlm.nih.gov/Blast.cgi) was used to search for sequences homologous to the 275 ORFs. A circular representation of the phage PF-CE2 genome was generated using the BLAST Ring Image Generator (BRIG) [14]. As shown in Fig. 1, the genome consisted of several clusters, including structural proteins, DNA replication and transcription 
proteins, nucleotide metabolism and biosynthesis proteins, lysis proteins, and DNA packaging proteins.

The above 275 ORFs were analyzed using the CAZy database (http://www.cazy.org/). The results showed that only the protein encoded by Gp190 was assigned to the GH family, which implies that Gp190 may have a function related to the degradation of exopolysaccharides. To validate this hypothesis, Gp190 was analyzed using PSI-BLAST (https://blast.ncbi.nlm.nih.gov/Blast.cgi) [15], and the results of the two iterations are shown in Table S4. In the first iteration, 33 homologous glycoside hydrolase family protein sequences were found in the "Sequence with E-values WORSE than the threshold". In the second iteration, 45 homologous sequences associated with glycoside hydrolase family proteins were shown in "Sequence with E-values BETTER than threshold". In addition, HHPred (https://toolkit.tuebingen.mpg.de/tools/hhpred) was also used to analyze the potential function of $G p 190$. The results showed that the N-terminus of Gp190 was similar to the glycoside hydrolase catalytic center of $1 \mathrm{WTH}$, with a probability of $100 \%$ [16], and that the C-terminus was similar to the glycoside hydrolase catalytic center of $3 \mathrm{~A} 1 \mathrm{M}$, with a probability of $94.4 \%$ [17]. Bacteriophage-borne glycanases are associated with the tail structure of many tailed phages. Phage $\mathrm{MMR} 11$ is a long-tailed phage that targets Staphylococcus aureus and protein Gp61, which has glycoside hydrolase activity, is located on the tail of this phage [18]. Phage P22 is a short-tailed phage and its tail spike protein, Gp26, has endorhamnosidase activity, which can specifically hydrolyze the Oantigen polysaccharide of Salmonella typhimurium [19]. K5 lyase A is also a tail spike protein and is encoded by the K5A coliphage, which is capable of degrading K5 exopolysaccharides with the repeat unit [-4)-GlcA-(1,4)-GlcNAc(1-] [20]. Here, Gp190 was also identified on the tail of phage PF-CE2. Therefore, according to the above analysis, there is a high possibility that Gp190 of phage PF-CE2 is capable of expressing bacteriophage-borne glycanase. Except for the Cronobacter phage vB CsaM leN, the other seven phages all contained a homologous sequence to Gp190 (>92\% identity, with 100\% coverage), suggesting that they may also express bacteriophage-borne glycanase. Regrettably, there have been few studies regarding the glycanases of $C$. sakazakii phages, so this study is beneficial for exploring which gene encodes glycanase and performing heterologous expression of this enzyme.

For further research and application of Gp190, several online tools were used to study its structure. The primary structure of Gp190 was analyzed using the ProtParam tool (https://web.expasy.org/protparam/) [21], which showed that Gp190 was 1761 bp, and encoded 586 amino acids. The molecular formula was $\mathrm{C}_{2821} \mathrm{H}_{4439} \mathrm{~N}_{797} \mathrm{O}_{895} \mathrm{~S}_{17}$, with a molecular weight of $64385.10 \mathrm{Da}$, a theoretical isoelectric point of 5.01, and a liposoluble index of 77.65. The instability index of Gp190 was predicted to be 29.77, which indicated 
that the protein was stable. The Phyre ${ }^{2}$ server

(http://www.sbg.bio.ic.ac.uk/phyre2/html/page.cgi?id=index) was utilized to predict the secondary structure of Gp190 [22] and showed that Gp190 contained 14\% alpha helices and 35\% beta strands. ProtScale (https://web.expasy.org/protscale/) was used to predict the hydrophilicity and hydrophobicity of the Gp190 protein [21] and revealed that hydrophilic amino acids accounted for $65 \%$, suggesting that Gp190 of phage PF-CE2 may be soluble. No transmembrane domain was identified in Gp190 using the TMHMM Server 2.0 (http://www.cbs.dtu.dk/services/TMHMM-2.0/). Additionally, Gp190 was determined to be non-secretory. Further, the SignalP 4.1 Server

(http://www.cbs.dtu.dk/services/SignalP-4.1/), and the PrediSi

(http://www.predisi.de/predisi/index.html) software were used to predict the signal peptide of Gp190, however no signal peptides were identified by either tool.

Although most proteins encoded by phage PF-CE2 were similar to the above-mentioned Cronobacter phages, there were still slight differences between them. For instance, Gp202 and Gp262 were unique genes to phage PF-CE2 (Fig. 1). Using HHPred software, Gp202 had a $96.23 \%$ possibility of encoding an $\mathrm{HNH}$ endonuclease, and the similarity between Gp202 and the mobile endonuclease, MobE encoded by Escherichia virus RB43, was 89\%. $\mathrm{HNH}$ endonuclease is an important assembly machine in the life cycle of bacteriophages. Most $\mathrm{HNH}$ endonucleases have DNA nicking activity, and MobE is believed to promote the movement of homing endonuclease I-TevIII, by cutting the specific non-coding region of the gene encoding the small subunits of aerobic ribonucleotide reductase (nrdB), which is conducive to the inheritance of homing endonuclease I-TevIII in the offspring phages [23, 24]. The protein encoded by Gp202 may be of great significance for the reproduction, and infection of phage PF-CE2. Gp261, Gp262, and Gp263 were all related to the synthesis of phage tail fibers. The possibility of Gp262 encoding long tail fibers was $98.25 \%$ according to HHPred software analysis. Enterobacteria phage T4 (NC_00866), also belonging to the subfamily Tevenvirinae of the family Myoviridae, contains both long- and short-tail fibers in its tail, which are responsible for host cell recognition and binding to the host cell. The long tail fiber of phage $\mathrm{T} 4$ recognizes outer membrane protein $\mathrm{C}$, or the lipopolysaccharides of host bacteria and is responsible for the initial and reversible attachment of the virion [25]. Therefore, Gp262 may be beneficial for phage PF-CE2 to recognize and adsorb host strain.

Previous studies have used the DNA polymerase and short tail fiber protein sequences to determine the phylogenetic relationships of several phages [26, 27]. The phylogenetic trees are shown in Fig. S1 and were made using MEGA 7.0 with the neighbor-joining tree method [28]. The tree based on the DNA polymerase, shows that phage PF-CE2 was grouped with the Cronobacter phage vB CsaM leB, suggesting a close relationship 
between these phages. In the short tail fiber protein tree, phage PF-CE2 was similar to the Citrobacter phage Margaery, with a bootstrap value of $98 \%$ and both grouped with a larger cluster, which included Cronobacter phages vB CsaM leE and vB CsaM leB. The Bacterial and Archaeal Viruses Subcommittee (BAVS) defined that the difference between two viruses belonging to the same species should be less than $5 \%$ at the nucleotide level. Recently, average nucleotide identity (ANI) has been used to assess the genetic relationships among species at the genome level [29-31]. ANI values of genus and species demarcation boundaries have a mean of $73.98 \%$ and $95 \%$, respectively [30, 31]. In addition, the ANI value based on more than 50\% coverage of genomes is credible [32]. Here we used JSpeciesWS (http://jspecies.ribohost.com/jspeciesws/) to estimate the ANI values between the phage PF-CE2 genome and other phage genomes [33]. When compared with phage genomes in 14 genera of the subfamily Tevenvirinae of the family Myoviridae, as shown in Fig. 2A and Table S5, phage PF-CE2 shared 88.65\% genome ANI with Escherichia phage RB16 (Escherichia virus RB16 genus, HM134276), which suggests that phage PF-CE2 belongs to the Escherichia virus RB16 genus. As shown in Fig. 2B and Table S6, phage PF-CE2 and the eight phages mentioned above, shared $<95 \%$ genome ANI with Escherichia phage RB16, but shared $>95 \%$ genome ANI with each other. This indicates that the nine phages could be assigned to a new species under the Escherichia virus RB16 genus of the subfamily Tevenvirinae in the family Myoviridae. There, we would name this new species, Cronobacter virus PF-CE2.

In conclusion, we determined the genome sequence of phage PF-CE2, belonging to the subfamily Tevenvirinae of the family Myoviridae. This finding is beneficial for identifying the gene encoding the bacteriophage-borne glycanase of the $C$. sakazakii phage, PF-CE2 and for understanding the mechanism of infection of phage PF-CE2 in host bacteria containing FcEPSs, which could promote the wide application of bacteriophage-borne glycanases in the preparation of fucosylated oligosaccharides. Moreover, a new species named Cronobacter virus PF-CE2 was established and the taxonomic status of eight phages was modified according to ANI analysis, which provides an important reference for the preservation and sharing of these phages.

\section{Declarations}

Nucleotide sequence accession number

The genome sequence of phage PF-CE2 was deposited in the GenBank database under the accession number MW629017.

Conflicts of interest 
The authors have declared no conflicts of interest.

\section{Funding}

The authors are grateful for the financial support by the National Natural Science Foundation of China (31872893).

\section{Ethical approval}

This article does not contain any studies with human participants or animals requiring ethical approval.

\section{References}

1. Endersen L, Buttimer C, Nevin E, Coffey A, Neve H, Oliveira H, Lavigne R, O'Mahony J (2017) Investigating the biocontrol and anti-biofilm potential of a three phage cocktail against Cronobacter sakazakii in different brands of infant formula. Int J Food Microbiol 253:1-11

2. Friedemann M (2007) Enterobacter sakazakii in food and beverages (other than infant formula and milk powder). Int J Food Microbiol 116:1-10

3. Drudy D, Mullane NR, Quinn T, Wall PG, Fanning S (2006) Enterobacter sakazakii: an emerging pathogen in powdered infant formula. Clin Infect Dis 42(7):996-1002

4. Healy B, Cooney S, O'Brien S, Iversen C, Whyte P, Nally J, Callanan JJ, Fanning S (2010) Cronobacter (Enterobacter sakazakii): an opportunistic foodborne pathogen. Foodborne Pathog Dis 7(4):339-350

5. McMinn A, Liang Y, Wang M (2020) Minireview: The role of viruses in marine photosynthetic biofilms. Mar Life Sci Technol 2:203-208

6. Zeng HY, He WJ, Li CS, Zhang JM, Li N, Ding Y, Xue L, Chen MT, Wu HM, Wu QP (2019) Complete genome analysis of a novel phage GW1 lysing Cronobacter. Arch Virol 164(2):625-628

7. Vanhooren P T, Vandamme E J (1999) L-fucose: occurrence, physiological role, chemical, enzymatic and microbial synthesis. J Chem Technol Biot 74(6):479-497

8. Elsässer-Beile U, Friebolin H, Stirm S (1978) Primary structure of Klebsiella serotype 6 capsular polyssacharide. Carbohydr Res 65(2):245-249

9. Xiao MS, Fu XD, Wei XY, Chi YZ, Gao W, Yu Y, Liu ZM, Zhu CL, Mou HJ (2021) Structural characterization of fucose-containing disaccharides prepared from exopolysaccharides of Enterobacter sakazakii. Carbohydr Polym 252:117139

10. Mcdermott JR, Shao QY, O'Leary C, Kongari R, Liu M (2019) Complete Genome Sequence of Citrobacter freundii Myophage Maroon. Microbiol Resour Announc 8(43):e01426-14

11. Abbasifar R, Kropinski AM, Sabour PM, Ackermann HW, Lingohr EJ, Griffiths MW (2012) Complete genome sequence of Cronobacter sakazakii bacteriophage vB_CsaM_GAP161. J Virol 86(24):13806-13807

12. Schattner P, Brooks AN, Lowe TM (2005) The tRNAscan-SE, snoscan and snoGPS web servers for the detection of tRNAs and snoRNAs. Nucleic Acids Res 33:W686-W689 
13. Lagesen K, Hallin P, Rodland EA, Staerfeldt HH, Rognes T, Ussery DW (2007) RNAmmer: consistent and rapid annotation of ribosomal RNA genes. Nucleic Acids Res 35:3100-3108

14. Alikhan, NF, Petty, NK, Zakour B, Beatson SA (2011) BLAST Ring Image Generator (BRIG): simple prokaryote genome comparisons. BMC Genomics 12:402

15. Shang AQ, Liu Y, Wang JL, Mo ZL, Li GY, Mou HJ (2015) Complete nucleotide sequence of Klebsiella phage P13 and prediction of an EPS depolymerase gene. Virus Genes 50(1):118-128.

16. Kanamaru S, Ishiwata Y, Suzuki T, Rossmann MG, Arisaka F (2005) Control of bacteriophage T4 tail lysozyme activity during the infection process. J Mol Biol 346(4):1013-1020.

17. Yokoi N, Inaba H, Terauchi M, Stieg AZ, Sanghamitra NJM, Koshiyama T, Yutani K, Kanamaru S, Arisaka F, Hikage T (2010) Construction of robust bio-nanotubes using the controlled self-assembly of component proteins of bacteriophage T4. Small 6(17):1873-1879.

18. Rashel M, Uchiyama J, Takemura I, Hoshiba H, Ujihara T, Takatsuji H, Honke K, Matsuzaki S (2008) Tail-associated structural protein Gp61 of Staphylococcus aureus phage phi MRU has bifunctional lytic activity. Fems Microbiol Lett 284(1):9-16

19. Andres D, Hanke C, Baxa U, Seul A, Seckler R (2010) Tailspike interactions with lipopolysaccharide effect DNA ejection from phage P22 particles in vitro. J Biol Chem 285(47):36768-36775

20. Thompson JE, Pourhossein M, Waterhouse A, Hudson T, Goldrick M, Derrick JP, Roberts IS (2010) The $\mathrm{K} 5$ lyase KflA combines a viral tail spike structure with a bacterial polysaccharide lyase mechanism. J Biol Chem 285(31):23963-23969.

21. Gasteiger E, Hoogland C, Gattiker A, Duvaud S, Wilkins MR, Appel RD, Bairoch A (2005) Protein identification and analysis tools on the ExPASy Server. In: Walker JM (eds): The proteomics protocols handbook. Springer protocols handbooks. Humana Press 571-607

22. Kelley LA, Mezulis S, Yates CM, Wass MN, Sternberg MJE (2015) The Phyre2 web portal for protein modeling, prediction and analysis. Nat Protoc 10(6):845-858

23. Zhang L, Xu D, Huang Y, Zhu X, Rui M, Wan T (2017). Structural and functional characterization of deep-sea thermophilic bacteriophage GVE2 HNH endonuclease. Sci Rep 7:42542

24. Wilson GW, Edgell D (2009) Phage T4 mobE promotes trans homing of the defunct homing endonuclease I-TevIII. Nucleic Acids Res 37:7110-7123

25. Meritxell G, Mikiyoshi N, Sara A, Shuji K, Mark VR (2017) Crystallization of the carboxy-terminal region of the bacteriophage T4 proximal long tail fibre protein gp34. Viruses 9(7):970.

26. Sváb D, Falgenhauer L, Rohde M, Szabó J, Chakraborty T, Tóth I (2018) Identification and Characterization of T5-Like Bacteriophages Representing Two Novel Subgroups from Food Products. Front Microbiol 9:202

27. Senevirathne A, Ghosh K, Roh E, Kim KP (2017) Complete genome sequence analysis of a novel Staphylococcus phage StAP1 and proposal of a new species in the genus Silviavirus. Arch Virol 162(7):2145-2148 
28. Kumar S, Stecher G, Tamura K (2016) MEGA7: molecular evolutionary genetics analysis version 7.0 for bigger datasets. Mol Biol Evol 33(7):1870-1874

29. Pacífico C, Hilbert M, Sofka D, Dinhopl N, Hilbertet F (2019) Natural occurrence of Escherichia coliinfecting bacteriophages in clinical samples. Front Microbiol 10:2484

30. Jain C, Rodriguez-R LM, Phillippy AM, Konstantinidis KT, Aluru S (2018) High throughput ANI analysis of $90 \mathrm{~K}$ prokaryotic genomes reveals clear species boundaries. Nat Commun 9:5114

31. Barco RA, Garrity GM, Scott JJ, Amend JP, Nealson KH, Emersonet D (2020) A genus definition for bacteria and archaea based on a standard genome relatedness index. mBio 11(1):e02475-19

32. Paepe MD, Hutinet G, Son O, Amarir-Bouhram J, Schbath S, Petit MA, Casadesús J (2014) Temperate phages acquire DNA from defective prophages by relaxed homologous recombination: the role of rad52-like recombinases. PLoS Genet 10(3):e1004181

33. Richter M, Rosselló-Móra R, Ckner F, Peplies J (2015) JSpeciesWS: a web server for prokaryotic species circumscription based on pairwise genome comparison. Bioinformatics, 32(6):929-931

\section{Figures}



GC Content GC Skew

ac sinem(-)

ac sinem(*)

Citrobacter_phage_Marcon

$100 \%$ identoty

706 identy

50s idenoty

Cronobacter_phage_vB_CsaM_Cronuts

1000 idertity

70s identy

50N ideraty

Cronobacter_phage_vB_CsaM_GAP161

$100 \mathrm{~s}$ identory

Tos ideraty

Dos identy

Cronobacter_phage_vB_CsaM_leB

[11000 identity

70s identy

In 50 s identy

Cronobacter_phage_vB_CsaM_leE

III $100 \mathrm{~s}$ ideresty

Til ros identy

III sos identy

Cronobacter_phage_vB_CsaM_leN

DI $100 \%$ identity

[11 ros identy

[1] sos idersty

Enterobacter_phage_vB_EkoM5VN

1000 identoty

ros identy

Insos identy

\section{Figure 1}


Schematic representation of the genomic organization of phage PF-CE2 compared to the eight other selected phages. The outermost circle represents the predicted protein functions of coding sequences: structural proteins (blue); replication and transcription (orange); DNA nucleotide metabolism and biosynthesis (yellow); lysis proteins (purple); DNA packaging (black); others (fuchsia).
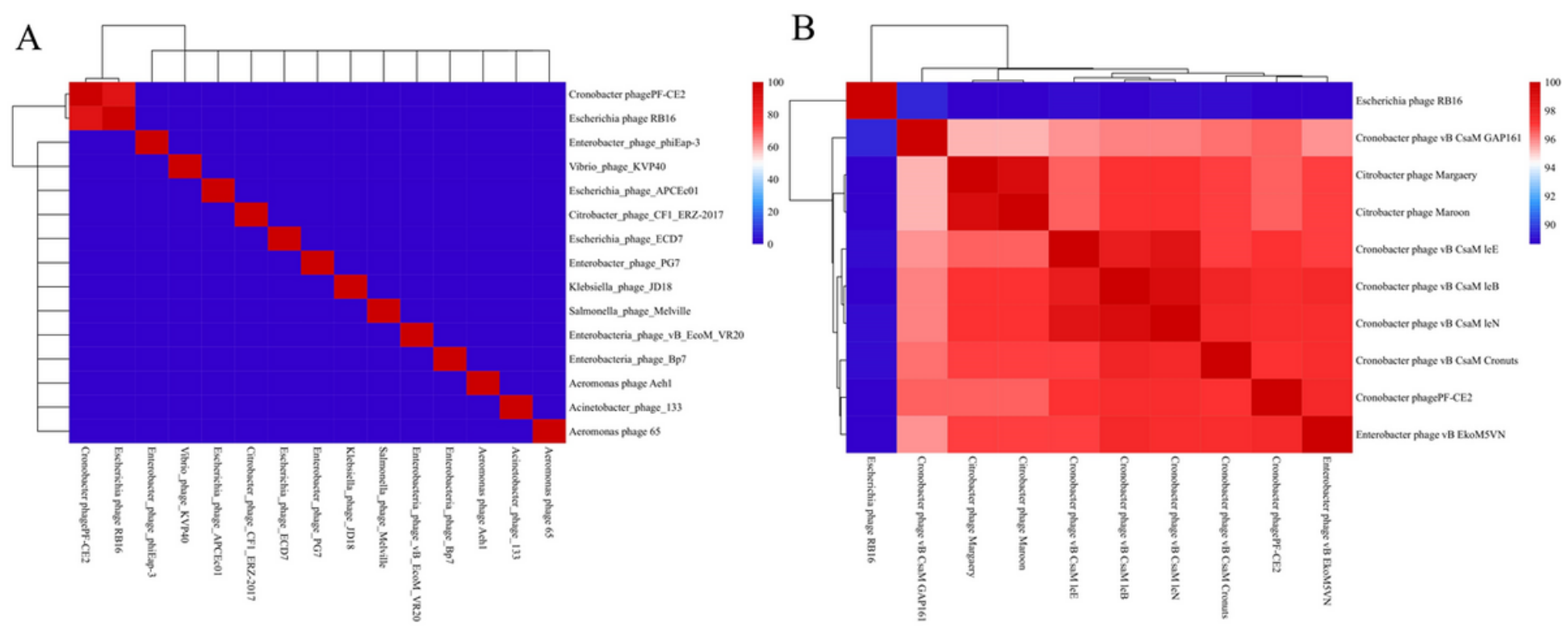

\section{Figure 2}

Heatmap showing the average nucleotide identity values determined for phage genomes. (A) Fourteen phage genomes from 14 genera of the subfamily Tevenvirinae of family Myoviridae were compared with the PF-CE2 phage genome, including Acinetobacter_phage_133 (Acinetobacter virus 133 genus, NC_015250); Aeromonas phage 65 (Aeromonas virus 65 genus, NC_015251); Aeromonas phage Aeh1 (Aeromonas virus Aeh1 genus, NC_005260); Enterobacteria_phage_Bp7 (Dhakavirus genus, NC_019500); Escherichia phage RB16 (Escherichia virus RB16 genus, HM134276);

Enterobacteria_phage_vB_EcoM_VR20 (Gaprivervirus genus, NC_028894); Salmonella_phage_Melville (Gelderlandvirus genus, NC_042044); Klebsiella_phage_JD18 (Jiaodavirus genus, NC_028686); Enterobacter_phage_PG7 (Karamvirus genus, NC_023561); Escherichia_phage_ECD7 (Krischvirus genus, NC_041936); Citrobacter_phage_CF1_ERZ-2017 (Moonvirus genus, NC_042067);

Escherichia_phage_APCEc01 (Mosigvirus genus, NC_029091); Vibrio_phage_KVP40 (Schizotequatrovirus genus, NC_005083); Enterobacter_phage_phiEap-3 (Slopekvirus genus, NC_041980). (B) The genomes of phage PF-CE2 and eight phages showing a high percentage of genome similarity to PF-CE2 were compared with the genome of Escherichia phage RB16.

\section{Supplementary Files}

This is a list of supplementary files associated with this preprint. Click to download.

- SupplementaryMaterials.doc 